\title{
Decorated Linear Order Types and the Theory of Concatenation
}

\author{
Vedran Čačić \\ Department of Mathematics \\ University of Zagreb \\ Bijenička 30, 10000 Zagreb \\ Croatia \\ veky@math.hr

\section{Greg Restall} \\ Department of Philosophy \\ The University of Melbourne \\ Parkville 3010 \\ Australia \\ restall@unimelb.edu.au \\ Pavel Pudlák \\ Mathematical Institute \\ Academy of Sciences of the Czech Republic \\ Žitná 25, 11567 Praha 1 \\ Czech Republic \\ pudlak@math.cas.cz \\ Alasdair Urquhart \\ Department of Philosophy \\ University of Toronto \\ 215 Huron Street, Toronto \\ Canada \\ urquhart@cs.toronto.edu \\ Albert Visser \\ Department of Philosophy \\ Utrecht University \\ Heidelberglaan 8, 3584 CS Utrecht \\ The Netherlands \\ albert.visser@phil.uu.nl
}

October 4, 2007

\begin{abstract}
We study the interpretation of Grzegorczyk's Theory of Concatenation TC in structures of decorated linear order types satisfying Grzegorczyk's axioms. We show that TC is incomplete for this interpretation. What is more, the first order theory validated by this interpretation interprets arithmetical truth. We also show that every extension of TC has a model that is not isomorphic to a structure of decorated order types.

We provide a positive result, to wit a construction that builds structures of decorated order types from models of a suitable concatenation theory. This construction has the property that if there is a representation of a certain kind, then the construction provides a representation of that kind.
\end{abstract}




\section{Introduction}

In his paper [Grz05], Andrzej Grzegorczyk introduces a theory of concatenation TC. The theory has a binary function symbol $*$ for concatenation and two constants a and $\mathrm{b}$. The theory is axiomatized as follows.

TC1. $\vdash(x * y) * z=x *(y * z)$

$\mathrm{TC2} . \vdash x * y=u * v \rightarrow((x=u \wedge y=v) \vee$ $\exists w((x * w=u \wedge y=w * v) \vee(x=u * w \wedge y * w=v)))$

TC3. $\vdash x * y \neq \mathrm{a}$

TC4. $\vdash x * y \neq \mathrm{b}$

TC5. $\vdash \mathrm{a} \neq \mathrm{b}$

Axioms TC1 and TC2 are due to Tarski. Grzegorczyk calls axiom TC2: the editor axiom. We will consider two weaker theories. The theory $\mathrm{TC}_{0}$ has the signature with just concatenation, and is axiomatized by $\mathrm{TC} 1,2$. The theory $\mathrm{TC}_{1}$ is axiomatized by $\mathrm{TC} 1,2,3$. We will also use $\mathrm{TC}_{2}$ for $\mathrm{TC}$.

The theories we are considering have various interesting interpretations. First they are, of course, theories of strings with concatenation. I.o.w., they are theories of free semigroups. Secondly they are theories of wider classes of structures, to wit structures of decorated linear order types, which will be defined below.

The theories $\mathrm{TC}_{i}$ are theories for concatenation without the empty string, i.e., without the unit element. Adding a unit $\varepsilon$ one obtains another class of theories $\mathrm{TC}_{i}^{\varepsilon}$, theories of free monoids, or theories of structures of decorated linear order types including the empty linear decorated order type. The basic list of axioms is as follows.

$\mathrm{TC}^{\varepsilon} 1 . \vdash \varepsilon * x=x \wedge x * \varepsilon=x$

$\mathrm{TC}^{\varepsilon} 2 . \vdash(x * y) * z=x *(y * z)$

$\mathrm{TC}^{\varepsilon} 3 . \vdash x * y=u * v \rightarrow \exists w((x * w=u \wedge y=w * v) \vee(x=u * w \wedge y * w=v))$

$\mathrm{TC}^{\varepsilon} 4 . \vdash \mathrm{a} \neq \varepsilon$

$\mathrm{TC}^{\varepsilon} 5 . \vdash x * y=\mathrm{a} \rightarrow(x=\varepsilon \vee y=\varepsilon)$

$\mathrm{TC}^{\varepsilon} 6 . \vdash \mathrm{b} \neq \varepsilon$

$\mathrm{TC}^{\varepsilon} 7 . \vdash x * y=\mathrm{b} \rightarrow(x=\varepsilon \vee y=\varepsilon)$

$\mathrm{TC}^{\varepsilon} 8 . \vdash \mathrm{a} \neq \mathrm{b}$

We take $\mathbf{T C}_{0}^{\varepsilon}$ to be the theory axiomatized by $\mathrm{TC}^{\varepsilon} 1,2,3$. We take $\mathrm{TC}_{1}^{\varepsilon}$ to be $\mathrm{TC}_{0}^{\varepsilon}+\mathrm{TC}^{\varepsilon} 4,5$ and $\mathrm{TC}^{\varepsilon}:=\mathrm{TC}_{2}^{\varepsilon}$ to be $\mathrm{TC}_{1}^{\varepsilon}+\mathrm{TC}^{\varepsilon} 6,7,8$. 
One can show that $\mathrm{TC}$ is bi-interpretable with $\mathrm{TC}^{\varepsilon}$, in which a unit $\varepsilon$ is added via one dimensional interpretations without parameters. ${ }^{1}$ The theory $\mathrm{TC}_{1}$ is biinterpretable with $\mathrm{TC}_{1}^{\varepsilon}$ via two-dimensional interpretations with parameters. The situation for $\mathrm{TC}_{0}$ seems to be more subtle. See also [Vis07]. In Section 6, we will study an extension of $\mathrm{TC}_{0}^{\varepsilon}$.

Andrzej Grzegorczyk and Konrad Zdanowski have shown that TC is essentially undecidable. This result can be strengthened by showing that Robinson's Arithmetic $Q$ is mutually interpretable with $T C$. Note that $T C_{0}$ is undecidable - since it has an extension that parametrically interprets $\mathrm{TC}$ - but that $\mathrm{TC}_{0}$ is not essentially undecidable: it is satisfied by a one-point model. Similarly $\mathrm{TC}_{1}$ is undecidable, but it has as an extension the theory of finite strings of a's, which is a notational variant of Presburger Arithmetic and, hence decidable.

We will call models of $\mathrm{TC}_{0}$ concatenation structures, and we will call models of $\mathrm{TC}_{i}$ concatenation $i$-structures. The relation of isomorphism between concatenation structures will be denoted by $\cong$. We will be interested in concatenation structures, whose elements are decorated linear order types with the operation concatenation of decorated order types. Let a non-empty class $A$ be given. An $A$-decorated linear ordering is a structure $\langle D, \leq, f\rangle$, where $D$ is a non-empty domain, $\leq$ is a linear ordering on $D$, and $f$ is a function from $D$ to $A$. A mapping $\bar{\phi}$ is an isomorphism between $A$-decorated linear order types $\langle D, \leq, f\rangle$ and $\left\langle D^{\prime}, \leq^{\prime}, f^{\prime}\right\rangle$ iff it is a bijection between $D$ and $D^{\prime}$ such that, for all $d, e$ in $D$, we have $d \leq e \Leftrightarrow \phi d \leq^{\prime} \phi e$, and $f d=f^{\prime} \phi d$. Our notion of isomorphism gives us a notion of $A$-decorated linear order type. We have an obvious notion of concatenation between $A$-decorated linear orderings which induces a corresponding notion of concatenation for $A$-decorated linear order types. We use $\alpha, \beta, \ldots$ to range over such linear order types. Since, linear order types are classes we have to follow one of two strategies: either to employ Scott's trick to associate a set object to any decorated linear order type or to simply refrain from dividing out isomorphism but to think about decorated linear orderings modulo isomorphism. We will employ the second strategy.

We will call a concatenation structure whose domain consists of (representatives of) $A$-decorated order types, for some $A$, and whose concatenation is concatenation of decorated order types: a concrete concatenation structure. It seems entirely reasonable to stipulate that e.g. the interpretation of a in a concrete concatenation structure is a decorated linear order type of a one element order. However, for the sake of generality we will refrain from making this stipilation.

Grzegorczyk conjectured that every concatenation 2-structure is isomorphic to a concrete concatenation structure. We prove that this conjecture is false. (i) Every extension of $\mathrm{TC}_{1}$ has a model that is not isomorphic to a concatenation 1-structure and (ii) the set of principles valid in all concrete concatenation 2structures interprets arithmetical truth.

\footnotetext{
${ }^{1}$ Albert Visser thinks he can improve this to: $\mathrm{TC}$ and $\mathrm{TC}^{\varepsilon}$ are definitionally equivalent.
} 
The plan of the paper is as follows. We show, in Section 2, that we have, for all decorated order types $\alpha, \beta$ and $\gamma$, the following principle:

$$
\text { (†) } \beta * \alpha * \gamma=\alpha \Rightarrow \beta * \alpha=\alpha * \gamma=\alpha .
$$

This fact was already known. It is due to Lindenbaum, credited to him in Sierpiński's book [Sie58] on p. 248. It is also problem 6.13 of [KT06]. Our proof, however, is different.

It is easy to see that every group is a concatenation structure and that $(\dagger)$ does not hold in the two element group. We show, in Section 5, that every concatenation structure can be extended to a concatenation structure with any number of atoms. It follows that there is a concatenation structure with at least two atoms in which ( $\dagger$ ) fails. Hence, TC is incomplete for concrete concatenation structures. In Section 3, we provide a counterargument of a different flavour. We provide a tally interpretation that defines the natural numbers (with concatenation in the role of addition) in every concrete concatenation 2 -structure. It follows that for every extension of $\mathrm{TC}_{1}$ is satisfied by a concatenation 1-structure that is not isomorphic to any concrete concatenation 1-structure, to wit any model of that extension that contains a non-standard element. In Section 4, we strengthen the result of Section 3, by showing that in concrete concatenation 2-structures we can add multiplication to the natural numbers. It follows that the set of arithmetically true sentences is interpretable in the concretely valid consequences of $\mathrm{TC}_{2}$.

Finally, in Section 6 we prove a positive result. We provide a mapping from aribitrary models of a variant of an extension of $\mathrm{TC}_{0}$ to structures of decorated order types. As we have shown such a construction cannot always provide a representation. We show that, for a restricted class of representations, we do have: if a model has a representation in the class, then the construction yields such a representation.

\section{Acknowledgments}

Some of the results of this note were obtained during the Excursion to mountain Ślęża of the inspiring Logic Colloquium 2007 in Wrocław and, in part, in the evening after the Excursion. We thank the organizers for providing this wonderful opportunity.

We thank Dana Scott for his comments, insights and questions. We are grateful to Vincent van Oostrom for some perceptive remarks.

Pavel Pudlák was supported by grants A1019401 and 1M002162080.

\section{A Principle for Decorated Order Types}

In this section we prove a universal principe that holds in all concatenation structures, which is not provable in TC. There is an earlier proof of this principle. 
See: [KT06], problem 6.13. Our proof, however, is different.

Theorem 2.1 Let $\alpha_{0}, \alpha_{1}, \alpha_{2}$ be decorated order types. Suppose $\alpha_{1}=\alpha_{0} * \alpha_{1} * \alpha_{2}$. Then $\alpha_{1}=\alpha_{0} * \alpha_{1}=\alpha_{1} * \alpha_{2}$.

\section{Proof}

Suppose $\alpha_{1}=\alpha_{0} * \alpha_{1} * \alpha_{2}$. Consider a decorated linear ordering $\mathcal{A}:=\langle A, \leq, f\rangle$ of type $\alpha_{1}$, By our assumption, we may partition $A$ into $A_{0}, A_{1}, A_{2}$, such that:

$$
\langle A, \leq, f\rangle=\left\langle A_{0}, \leq \uparrow A_{0}, f\left\lceil A_{0}\right\rangle *\left\langle A_{1}, \leq \uparrow A_{1}, f\left\lceil A_{1}\right\rangle *\left\langle A_{2}, \leq \uparrow A_{2}, f \uparrow A_{2}\right\rangle,\right.\right.
$$

where $\mathcal{A}_{i}:=\left\langle A_{i}, \leq\left\lceil A_{i}, f\left\lceil A_{i}\right\rangle\right.\right.$ is an instance of $\alpha_{i}$, Let $\phi: \mathcal{A} \rightarrow \mathcal{A}_{1}$ be an isomorphism.

Let $\phi^{n} \mathcal{A}_{(i)}:=\left\langle\phi^{n}\left[A_{(i)}\right], \leq\left\lceil\phi^{n}\left[A_{(i)}\right], f\left\lceil\phi^{n}\left[A_{(i)}\right]\right\rangle\right.\right.$. We have: $\phi^{n} \mathcal{A}_{i}$ is of order type $\alpha_{i}$ and $\phi^{n} \mathcal{A}$ is of order type $\alpha_{1}$.

Clearly, $\phi \mathcal{A}_{0}$ is an initial substructure of $\phi \mathcal{A}=\mathcal{A}_{1}$. So, $\mathcal{A}_{0}$ and $\phi \mathcal{A}_{0}$ are disjoint and $\phi \mathcal{A}_{0}$ adjacent to the right of $\mathcal{A}_{0}$. Similarly, for $\phi^{n} \mathcal{A}_{0}$ and $\phi^{n+1} \mathcal{A}_{0}$. Take $A_{0}^{\omega}:=\bigcup_{i \in \omega} \phi^{i} A_{0}$. We find that $\mathcal{A}_{0}^{\omega}:=\left\langle A_{0}^{\omega}, \leq \uparrow A_{0}^{\omega}, f \uparrow A_{0}^{\omega}\right\rangle$ is initial in $\mathcal{A}$ and of decorated linear order type $\alpha_{0}^{\omega}$. So $\alpha_{1}=\alpha_{0}^{\omega} * \rho$, for some $\rho$. It follows that $\alpha_{0} * \alpha_{1}=\alpha_{0} * \alpha_{0}^{\omega} * \rho=\alpha_{0}^{\omega} * \rho=\alpha_{1}$. The other identity is similar.

So, every concrete concatenation structure validates that $\alpha_{1}=\alpha_{0} * \alpha_{1} * \alpha_{2}$ implies $\alpha_{1}=\alpha_{0} * \alpha_{1}=\alpha_{1} * \alpha_{2}$. We postpone the proof that this principle is not provable in TC to Section 5.

\section{Definability of the Natural Numbers}

In this section, we show that the natural numbers can be defined in every concrete concatenation 1-structure. We define:

- $x \subseteq y: \leftrightarrow x=y \vee \exists u(u * x=y) \vee \exists v(x * v=y) \vee \exists u, v(u * x * v=y)$.

- $x \subseteq_{\text {ini }} y: \leftrightarrow x=y \vee \exists v(x * v=y)$.

- $x \subseteq_{\text {end }} y: \leftrightarrow x=y \vee \exists u(u * x=y)$.

- $\left(n: \widetilde{\mathrm{N}}_{\mathrm{a}}\right): \leftrightarrow \forall m \subseteq_{\text {ini }} n(m=\mathrm{a} \vee \exists k(k \neq m \wedge m=k * \mathrm{a}))$.

The use of ' $:$ ' in $n: \widetilde{N}_{\mathrm{a}}$ is derived from the analogous use in type theory. We could read it as: $n$ is of sort $\mathrm{N}_{\mathrm{a}}$. We write $m, n: \widetilde{\mathrm{N}}_{\mathrm{a}}$ for: $\left(m: \widetilde{\mathrm{N}}_{\mathrm{a}}\right) \wedge\left(n: \widetilde{\mathrm{N}}_{\mathrm{a}}\right)$. Etc. In the context of a structure we will confuse $\widetilde{N}_{a}$ with the extension of $\widetilde{\mathrm{N}}_{\mathrm{a}}$ in that structure.

We prove the main theorem of this section. 
Theorem 3.1 In any concrete concatenation structure, we have:

$$
\widetilde{\mathrm{N}}_{\mathrm{a}}=\left\{\mathrm{a}^{n+1} \mid n \in \omega\right\} .
$$

I.o.w, $\widetilde{\mathrm{N}}_{\mathrm{a}}$ is precisely the class of natural numbers in tally representation (starting with 1). Note that $*$ on this set is addition.

\section{Proof}

Consider any concrete concatenation 1-structure $\mathfrak{A}$. It is easy to see that every $\mathrm{a}^{n+1}$ is in $\widetilde{\mathrm{N}}_{\mathrm{a}}$.

Clearly, every element $x$ of $\widetilde{N}_{\mathrm{a}}$ is either a or it has a predecessor, i.e., there is a $y$ such that $x=y * \mathrm{a}$. The axioms of $\mathrm{TC}_{1}$ guarantee that this predecessor is unique. This justifies the introduction of the partial predecessor function pd on $\widetilde{N}_{\mathrm{a}}$. Let $\alpha$ be the order type corresponding to a. Let $\beta_{0}$ be any element of $\widetilde{N}_{\mathrm{a}}$. If, for some $n, \operatorname{pd}^{n} \beta_{0}$ is undefined, then $\beta_{0}$ is clearly of the form $\alpha^{k+1}$, for $k$ in $\omega$.

We show that the other possibility cannot obtain. Suppose $\beta_{n}:=\operatorname{pd}^{n} \beta_{0}$ is always defined. Let $\mathcal{A}$ be a decorated linear ordering of type $\alpha$ and let $\mathcal{B}_{i}$ be a decorated linear ordering of type $\beta_{i}$. We assume that the domain $A$ of $\mathcal{A}$ is disjoint from the domains $B_{i}$ of the $\mathcal{B}_{i}$. Thus, we may implement $\mathcal{B}_{i+1} * \mathcal{A}$ just by taking the union of the domains.

Let $\phi_{i}$ be isomorphisms from $\mathcal{B}_{i+1} * \mathcal{A}$ to $\mathcal{B}_{i}$. Let $\mathcal{A}_{i}:=\left(\phi_{0} \circ \cdots \circ \phi_{i}\right)(\mathcal{A})$. Then, the $\mathcal{A}_{i}$ are all of type $\alpha$ and, for some $\mathcal{C}$, we have $\mathcal{B}_{0} \cong \mathcal{C} * \cdots * \mathcal{A}_{1} * \mathcal{A}_{0}$. Similarly $\mathcal{B}_{1} \cong \mathcal{C} * \cdots * \mathcal{A}_{2} * \mathcal{A}_{1}$. Let $\breve{\omega}$ be the opposite ordering of $\omega$. It follows that $\beta_{0}=\gamma * \alpha^{\breve{\omega}}=\beta_{1}=\operatorname{pd}\left(\beta_{0}\right)$. Hence, $\beta_{0}$ is not in $\widetilde{N}_{\mathrm{a}}{ }^{2}$ A contradiction.

We call a concatenation structure standard if $\widetilde{\mathrm{N}}_{\mathrm{a}}$ defines the tally natural numbers. Since, by the usual argument, any any extension of $\mathrm{TC}_{1}$ has a model with non-standard numbers, we have the following corollary.

Corollary 3.2 Every extension of $\mathrm{TC}_{1}$ has a model that is not isomorphic to a concrete concatenation 1-structure. In a different formulation: for every concatenation 1-structure there is an elementarily equivalent concatenation 1-structure that is not isomorphic to a concrete concatenation 1-structure.

Note that the non-negative tally numbers with addition form a concrete concatenation 1-structure. Thus, the concretely valid consequences of $\mathrm{TC}_{1}+\forall x\left(x: \widetilde{\mathrm{N}}_{\mathrm{a}}\right)$, i.e., the principles valid in every concrete concatenation 1-structure satisfying $\forall x\left(x: \widetilde{N}_{\mathrm{a}}\right)$ are decidable.

\section{Definability of Multiplication}

If we have two atoms to work with, we can add multiplication to our tally numbers. This makes the set of concretely valid consequences of TC nonarithmetical. The main ingredient of the definition of multiplication is the

\footnotetext{
${ }^{2}$ Note that we are not assuming that $\gamma$ is in $\mathfrak{A}$.
} 
theory of relations on tally numbers. In TC, we can develop such a theory. We represent the relation $\left\{\left\langle x_{0}, y_{0}\right\rangle, \ldots,\left\langle x_{n-1}, y_{n-1}\right\rangle\right\}$, by:

$$
\mathrm{bb} * x_{0} * \mathrm{~b} * y_{0} * \mathrm{bb} * x_{1} * \ldots \mathrm{bb} * x_{n-1} * \mathrm{~b} * y_{n-1} * \mathrm{bb} .
$$

We define:

- $r: \mathrm{REL}: \leftrightarrow \mathrm{bb} \subseteq$ end $r$,

- $\emptyset:=\mathrm{bb}$,

- $x[r] y: \leftrightarrow x, y: \widetilde{\mathrm{N}}_{\mathrm{a}} \wedge \mathrm{bb} * x * \mathrm{~b} * y * \mathrm{bb} \subseteq r$.

- $\operatorname{adj}(r, x, y):=r * x * \mathrm{~b} * y * \mathrm{bb}$.

Clearly, we have: TC $\vdash \forall u, v \neg u[\emptyset] v$. To verify that this coding works we need the adjunction principle.

Theorem 4.1 We have:

$\mathrm{TC} \vdash\left(r: \operatorname{REL} \wedge x, y, u, v: \widetilde{\mathrm{N}}_{\mathrm{a}}\right) \rightarrow(u[\operatorname{adj}(r, x, y)] v \leftrightarrow(u[r] v \vee(u=x \wedge v=y)))$.

We can prove this result by laborious and unperspicuous case splitting. However, it is more elegant to do the job with the help of a lemma. Consider any model of $\mathrm{TC}_{0}$. Fix an element $w$. We call a sequence $\left(w_{0}, \ldots, w_{k}\right)$ a partition of $w$ if we have that $w_{0} * \cdots * w_{k}=w$. The partitions of $w$ form a category with the following morphisms. $f:\left(u_{0}, \ldots, u_{n}\right) \rightarrow\left(w_{0}, \ldots, w_{k}\right)$ iff $f$ is a surjective and weakly monotonic function from $n+1$ to $k+1$, such that, for any $i \leq k, w_{i}=$ $u_{s} * \cdots * u_{\ell}$, where $f(j)=i$ iff $s \leq j \leq \ell$. We write $\left(u_{0}, \ldots, u_{n}\right) \leq\left(w_{0}, \ldots, w_{k}\right)$ for: $\exists f f:\left(u_{0}, \ldots, u_{n}\right) \rightarrow\left(w_{0}, \ldots, w_{k}\right)$. In this case we say that $\left(u_{0}, \ldots, u_{n}\right)$ is a refinement of $\left(w_{0}, \ldots, w_{k}\right)$.

Lemma 4.1 Consider any concatenation structure. Let $w$ be an element of the structure. Then, any two partitions of $w$ have a common refinement.

\section{Proof}

Fix any concatenation structure. We first prove that, for all $w$, all pairs of partitions $\left(u_{0}, \ldots, u_{n}\right)$ and $\left(w_{0}, \ldots, w_{k}\right)$ of $w$ have a common refinement, by induction of $n+k$.

If either $n$ or $k$ is 0 , this is trivial. Suppose $\left(u_{0}, \ldots, u_{n+1}\right)$ and $\left(w_{0}, \ldots, w_{n+1}\right)$ are partitions of $w$. By the editor axiom, either (a) $u_{0} * \cdots * u_{n}=w_{0} * \cdots * w_{k}$ and $u_{n+1}=w_{k+1}$, or there is a $v$ such that (b) $u_{0} * \cdots * u_{n} * v=w_{0} * \cdots * w_{k}$ and $u_{n+1}=v * w_{k+1}$, or (c) $u_{0} * \cdots * u_{n}=w_{0} * \cdots * w_{k} * v$ and $v * u_{n+1}=$ $w_{k+1}$. We only treat case (b), the other cases being easier or similar. By the induction hypothesis, there is a common refinement $\left(x_{0}, \ldots x_{m}\right)$ of $\left(u_{0}, \ldots, u_{n}, v\right)$ and $\left(w_{0}, \ldots, w_{n}\right)$. Let this be witnessed by $f$, resp. $g$. It is easily seen that $\left(x_{0}, \ldots x_{m}, w_{k+1}\right)$ is the desired refinement with witnessing functions $f^{\prime}$ and $g^{\prime}$, where $f^{\prime}:=f[m+1 \mapsto n+1], g^{\prime}:=g[m+1 \mapsto k+1]$. Here $f[m+1 \mapsto n+1]$ in the result of extending $f$ to assign $n+1$ to $m+1$. 
We turn to the proof of Theorem 4.1. The verification proceeds more or less as one would do it for finite strings.

\section{Proof}

Consider any concatenation 2-structure. Suppose REL $(r)$. The right-to-left direction is easy, so we treat left-to-right. Suppose $x, y, u$ and $v$ are tally numbers. and $u[\operatorname{adj}(r, x, y)] v$. There are two possibilities. Either $r=\mathrm{bb}$ or $r=$ $r_{0} * \mathrm{bb}$. We will treat the second case. Let $s:=\operatorname{adj}(r, x, y)$. One the following four partitions is a partition of $s$ : (i) (b, b, $u, \mathrm{~b}, v, \mathrm{~b}, \mathrm{~b})$, or (ii) $(w, \mathrm{~b}, \mathrm{~b}, u, \mathrm{~b}, v, \mathrm{~b}, \mathrm{~b})$, or (iii) (b, b, $u, \mathrm{~b}, v, \mathrm{~b}, \mathrm{~b}, z)$, or (iv) $(w, \mathrm{~b}, \mathrm{~b}, u, \mathrm{~b}, v, \mathrm{~b}, \mathrm{~b}, z)$. We will treat cases (ii) and (iv).

Suppose $\sigma:=(w, \mathrm{~b}, \mathrm{~b}, u, \mathrm{~b}, v, \mathrm{~b}, \mathrm{~b})$ is a partition of $s$. We also have that $\tau:=$ $\left(r_{0}, \mathrm{~b}, \mathrm{~b}, x, \mathrm{~b}, y, \mathrm{~b}, \mathrm{~b}\right)$ is a partition of $s$. Let $\left(t_{0}, \ldots, t_{k}\right)$ be a common refinement of $\sigma$ and $\tau$, with witnessing functions $f$ and $g$. The displayed b's in these partitions must have unique places among the $t_{i}$. We define $m_{\sigma}$ to be the unique $i$ such that $f(i)=m$, provided that $\sigma_{m}=\mathrm{b}$. Similarly, for $m_{\tau}$. (To make this unambiguous, we assume that if $\sigma=\tau$, we take $\sigma$ as the common refinement with $f$ and $g$ both the identity function.)

We evidently have $7_{\sigma}=7_{\tau}=k$ and $6_{\sigma}=6_{\tau}=k-1$. Suppose $4_{\sigma}<4_{\tau}$. It follows that $\mathrm{b} \subseteq v$. So, $v$ would have an initial subsequence that ends in $\mathrm{b}$, which is impossible. So, $4_{\sigma} \nless 4_{\tau}$. Similarly, $4_{\tau} \nless 4_{\sigma}$. So $4_{\sigma}=4_{\tau}$. It follows that $v=y$. Reasoning as in the case of $4_{\sigma}$ and $4_{\tau}$, we can show that $2_{\sigma}=2_{\tau}$ and, hence $u=x$.

Suppose $\rho:=(w, \mathrm{~b}, \mathrm{~b}, u, \mathrm{~b}, v, \mathrm{~b}, \mathrm{~b}, z)$ is a partition of $s$. We also have that $\tau:=$ $\left(r_{0}, \mathrm{~b}, \mathrm{~b}, x, \mathrm{~b}, y, \mathrm{~b}, \mathrm{~b}\right)$ is a partition of $s$. Let $\left(t_{0}, \ldots, t_{k}\right)$ be a common refinement of $\rho$ and $\tau$, with witnessing functions $f$ and $g$. We consider all cases, where $1_{\tau}<6_{\rho}$. Suppose $6_{\rho}=1_{\tau}+1=2_{\tau}$. Note that $7_{\rho}=6_{\rho}+1$, so we find: $\mathrm{b} \subseteq x$, quod non, since $x$ is in $\widetilde{\mathrm{N}}_{\mathrm{a}}$. Suppose $2_{\tau}<6_{\rho}<4_{\tau}$. In this case we have a b as substring of $x$. Quod non. Suppose $6_{\rho}=4_{\tau}$. Since $7_{\rho}=6_{\rho}+1$, we get a $\mathrm{b}$ in $y$. Quod non. Suppose $4_{\tau}<6_{\rho}<6_{\tau}$. In this case, we get a b in $y$. Quod impossibile. Suppose $6_{\rho} \geq 6_{\tau}=k-1$. In this place there is no place left for $z$ among the $t_{i}$. So, in all cases, we obtain a contradiction. So the only possibility is $6_{\rho} \leq 1_{\tau}$. Thus, it follows that $u[r] v$.

We can now use our relations to define multiplication of tally numbers in the usual way. See e.g. Section 2.2 of [Bur05]. In any concrete concatenation 2structure, we can use induction to verify the defining properties of multiplication as defined. It follows that we can interpret all arithmetical truths in the set of concretely valid consequences of TC.

Corollary 4.2 We can interpret true arithmetic in the set of all principles valid in concrete concatenation 2-structures. 


\section{The Sum of Concatenation Structures}

In this section we show that concatenation structures are closed under sums. This result will make it possible to verify the claim that the universal principle of Section 2 is not provable in TC. The result has some independent interest, since it provides a good closure property of concatenation structures.

Consider two concatenation structures $\mathfrak{A}_{0}$ and $\mathfrak{A}_{1}$. We write $\star$ for concatenation in the $\mathfrak{A}_{i}$. We may assume, without loss of generality, that the domains of $\mathfrak{A}_{0}$ and $\mathfrak{A}_{1}$ are disjoint. We define the sum $\mathfrak{B}:=\mathfrak{A}_{0} \oplus \mathfrak{A}_{1}$ as follows.

- The domain of $\mathfrak{B}$ consists of non-empty sequences $w_{0} \cdots w_{n-1}$, where the $w_{j}$ are alternating between elements of the domains of $\mathfrak{A}_{0}$ and $\mathfrak{A}_{1}$. In other words, if $w_{j}$ is in the domain of $\mathfrak{A}_{i}$, then $w_{j+1}$, if it exists, is in the domain of $\mathfrak{A}_{1-i}$.

- The concatenation $\sigma * \tau$ of $\sigma:=w_{0} \cdots w_{n-1}$ and $\tau:=v_{0} \cdots v_{k-1}$ is $w_{0} \cdots w_{n-1} v_{0} \cdots w_{k-1}$, in case $w_{n-1}$ and $v_{0}$ are in the domains of different structures $\mathfrak{A}_{i}$. The concatenation $\sigma * \tau$ is $w_{0} \cdots\left(w_{n-1} \star v_{0}\right) \cdots w_{k-1}$, in case $w_{n-1}$ and $v_{0}$ are in in the same domain.

In case $\sigma * \tau$ is obtained via the first case, we say that $\sigma$ and $\tau$ are glued together. If the second case obtains, we say that $\sigma$ and $\tau$ are clicked together.

Theorem 5.1 The structure $\mathfrak{B}=\mathfrak{A}_{0} \oplus \mathfrak{A}_{1}$ is a concatenation structure.

\section{Proof}

Associativity is easy. We check the editor property TC2. Suppose $\sigma_{0} * \sigma_{1}=$ $z_{0} \cdots z_{m-1}=\tau_{0} * \tau_{1}$. We distinguish a number of cases.

Case 1. Suppose both of the pairs $\sigma_{0}, \sigma_{1}$ and $\tau_{0}, \tau_{1}$ are glued together. Then, for some $k, n>0$, we have $\sigma_{0}=z_{0} \cdots z_{k-1}, \sigma_{1}=z_{k} \cdots z_{m-1}, \tau_{0}=z_{0} \cdots z_{n-1}$, and $\tau_{1}=z_{n} \cdots z_{m-1}$.

So, if $k=n$, we have $\sigma_{0}=\tau_{0}$ and $\sigma_{1}=\tau_{1}$.

If $k<n$, we have $\tau_{0}=\sigma_{0} *\left(z_{k} \cdots z_{n-1}\right)$ and $\sigma_{1}=\left(z_{k} \cdots z_{n-1}\right) * \tau_{1}$. The case that $n<k$ is similar.

Case 2. Suppose $\sigma_{0}, \sigma_{1}$ is glued together and that $\tau_{0}, \tau_{1}$ is clicked together. So, there are $k, n>0, u_{0}$, and $u_{1}$ such that $\sigma_{0}=z_{0} \cdots z_{k-2} u_{0}, \sigma_{1}=u_{1} z_{k} \cdots z_{m-1}$, $u_{0} \star u_{1}=z_{k-1}, \tau_{0}=z_{0} \cdots z_{n-1}$, and $\tau_{1}=z_{n} \cdots z_{m-1}$.

Suppose $k \leq n$. Then, $\tau_{0}=\sigma_{0} *\left(u_{1} z_{k} \cdots z_{n-1}\right)$ and $\sigma_{1}=\left(u_{1} z_{k} \cdots z_{n-1}\right) * \tau_{1}$. Note that, in case $k=n$, the sequence $z_{k} \cdots z_{n-1}$ is empty. The case that $k \geq n$ is similar.

Case 3. This case, where $\sigma_{0}, \sigma_{1}$ is clicked together and $\tau_{0}, \tau_{1}$ is glued together, is similar to case 2 . 
Case 4. Suppose that $\sigma_{0}, \sigma_{1}$ and $\tau_{0}, \tau_{1}$ are both clicked together. So, there are $k, n>0, u_{0}, u_{1}, v_{0}, v_{1}$ such that $\sigma_{0}=z_{0} \cdots z_{k-2} u_{0}, \sigma_{1}=u_{1} z_{k} \cdots z_{m-1}$, $u_{0} \star u_{1}=z_{k-1}, \tau_{0}=z_{0} \cdots z_{n-2} v_{0}, \tau_{1}=v_{1} z_{n} \cdots z_{m-1}$ and $v_{0} \star v_{1}=z_{n-1}$.

Suppose $k=n$. We have $u_{0} \star u_{1}=z_{k-1}=v_{0} \star v_{1}$. So, we have either (a) $u_{0}=v_{0}$ and $u_{1}=v_{1}$, or, for some $w$, either (b) $u_{0} \star w=v_{0}$ and $u_{1}=w \star v_{1}$, or (c) $u_{0}=v_{0} \star w$ and $w \star u_{1}=v_{1}$. In case (b), we have: $\sigma_{0} * w=\tau_{0}$ and $\sigma_{1}=w * \tau_{1}$. We leave (a) and (c) to the reader.

Suppose $k<n$. We have:

$$
\sigma_{0} *\left(u_{1} z_{k} \cdots z_{n-2} v_{0}\right)=\tau_{0} \text { and } \sigma_{1}=\left(u_{1} z_{k} \cdots z_{n-2} v_{0}\right) * \tau_{1} .
$$

The case that $k>n$ is similar.

It is easy to see that $\oplus$ is a sum or coproduct in the sense of category theory. The following theorem is immediate.

Theorem 5.2 If $a$ is an atom of $\mathfrak{A}_{i}$, then a is an atom of $\mathfrak{A}_{0} \oplus \mathfrak{A}_{1}$.

Finally, we have the following theorem.

Theorem 5.3 Let $A$ be any set and let $\mathfrak{B}:=\langle B, *\rangle$ be any concatenation structure. We assume that $A$ and $B$ are disjoint. Then, there is an extension of $\mathfrak{B}$ with at least $A$ as atoms.

\section{Proof}

Let $A^{*}$ be the free semi-group generated by $A$. We can take as the desired extension of $\mathfrak{B}$, the structure $A^{*} \oplus \mathfrak{B}$.

Remark 5.4 The whole development extends with only minor adaptations, when we replace axiom TC2 by:

- $\vdash x * y=u * v \rightarrow((x=u \wedge y=v) \dot{\vee}(\exists ! w(x * w=u \wedge y=w * v) \vee$

$$
\exists ! w(x=u * w \wedge y * w=v))
$$

Here $\dot{\vee}$ is exclusive or.

\section{A Canonical Construction}

Although we know that not every concatenation structure can be represented by decorated linear orderings, i.e., as a concrete concatenation structure, there may exist a canonical construction of a concrete concatenation structure which is a representation whenever there exists any concrete representation. In this section we shall propose such a construction, but we can only show that it is universal in a restricted subclass of all concrete representations.

It will be now more convenient to work with a theory for monoids, rather than for semigroups, as we did in the previous sections. We will work in the theory $\mathrm{TC}_{0}^{\varepsilon}$ plus the following axiom. 
$\mathrm{TC}^{\varepsilon} 9 . \vdash x * y * z=y \rightarrow(x=\varepsilon \wedge z=\varepsilon)$.

We do not postulate the existence of irreducible elements, as they do not play any role in what follows, but they surely can be present. We shall call elements of a model $\mathcal{M}$ of $\mathrm{TC}_{!}^{\varepsilon}$ : words. When possible, the concatenation symbol $*$ will be omitted.

Lemma 6.1 In a model $\mathcal{M}$ of $\mathrm{TC}_{!}^{\varepsilon}$ the binary relation $\exists u(x u=y)$ defines an ordering on the elements of $\mathcal{M}$.

Definition 6.1 Let $w$ be a word.

- A $k$-partition of $w$ is a $k$-tuple $\left(w_{1}, \ldots, w_{k}\right)$ such that $w_{1} \ldots w_{k}=w$; we shall often abbreviate it by $w_{1} \ldots w_{k}$.

- An ordering relation is defined on 3-partitions of $w$ by

$$
u_{1} u_{2} u_{3} \leq v_{1} v_{2} v_{3} \equiv \exists x_{1}, x_{3}\left(v_{1} x_{1}=u_{1} \wedge x_{3} v_{3}=u_{3}\right) .
$$

The axioms ensure that for any two partitions there is a unique common refinement.

Definition 6.2 [Word Ultrafilters] Let $w$ be a word and $S$ a set of 3-partitions of $w$. We shall call $S$ a word ultrafilter (wuf) on $w$ if

1. $\varepsilon w \varepsilon \in S$

2. $x \varepsilon y \notin S$ for any $x, y$

3. if $U \in S, V$ is a 3-partition of $w$ and $U \leq V$, then $V \in S$

4. if $x y z \in S$ and $y=y_{1} y_{2}$ then exactly one of the following two cases holds: $\left(x, y_{1}, y_{2} z\right) \in S$ or $\left(x y_{1}, y_{2}, z\right) \in S$.

Let $S$ be a wuf on $w$ and $x y z \in S$. Then we define the natural restriction of $S$ to $y$ which is a wuf $S_{y}$ on $y$ defined by:

$$
(r, s, t) \in S_{y}: \Leftrightarrow(x r, s, t z) \in S .
$$

We shall define an ordering on wuf's on a fixed $w$ and an equivalence on wuf's on all words of $M$.

- Let $S$ and $T$ be wuf's on $w$, then we define

$$
S<T: \Leftrightarrow \exists u, v((\varepsilon, u, v) \in S \wedge(u, v, \varepsilon) \in T) .
$$


- Let $S$ and $T$ be wuf's on possibly different words, then we define

$$
S \sim T \Leftrightarrow \exists x, x^{\prime}, y, z, z^{\prime}\left(x y z \in S \wedge x^{\prime} y z^{\prime} \in T \wedge S_{y}=T_{y}\right) .
$$

Definition 6.3 Let $w$ be a word. The canonical decorated ordering associated with $w$ is the ordering of all wuf's on $w$, where each wuf $S$ is decorated by $[S]_{\sim}$, the equivalence class of $\sim$ containing $S$. This decorated ordering will be denoted by $C(w)$.

Here are some basic properties of $C(w)$.

- The topological space determined by the ordering is compact and totally disconnected. In particular, it has the largest and the smallest elements.

- For every proper prefix $x$ of $w$, there is a uniquely determined pair of wuf's which forms a gap (no wuf in between). Thus there is a natural embedding of the ordering of the prefixes into $C(w)$. More precisely, we have have two mappings $\phi_{w}^{-}$and $\phi_{w}^{+}$such that for a proper prefix $x$ the pair $\phi_{w}^{-}(x), \phi_{w}^{+}(x)$ is the gap corresponding to $x$. If $x=\varepsilon$ (or $x=w$ ) then only $\phi_{w}^{+}(x)$ (or $\phi_{w}^{-}(x)$ ) is defined and it is the least (largest) element of $C(w)$. Furthermore the images of these mappings are dense sets in $C(w)$.

- Vice versa, every gap in $C(w)$ corresponds to a prefix (or equivalently to a 2-partition).

- If $a$ is an atom (irreducible element in $\mathcal{M}$ ), then it determines a principal wuf. For a given atom $a$ all such principal wuf's are equivalent.

Definition 6.4 $\rho$ is a regular representation of $\mathcal{M}$ by decorated orderings, if for every 2-partition $x_{1} x_{2}=w$ of $w \in \mathcal{M}$, there exists a unique 2-partition $A_{1} A_{2}=\rho(w)$ such that $A_{1} \cong \rho\left(x_{1}\right)$ and $A_{2} \cong \rho\left(x_{2}\right)$.

We do not know if every concatenation structure that has a concrete represntation also has a concrete regular representation.

If $\rho$ is regular, we have an analogous property for $k$-partitions for every $k$. For a $k$-partition $\left(x_{1}, \ldots, x_{k}\right)$ of $w$ in $\mathcal{M}$, we shall write

$$
\rho^{k}\left(x_{1}, \ldots, x_{k}\right)=\left(A_{1}, \ldots, A_{k}\right),
$$

where $\left(A_{1}, \ldots, A_{k}\right)$ is the uniquely determined $k$-partition of $\rho\left(x_{1} \ldots x_{k}\right)$ such that $A_{i} \cong \rho\left(x_{i}\right)$, for $i=1, \ldots, k$.

Theorem 6.5 1. If the canonical mapping $C$ is a representation of $\mathcal{M}$, then it is a regular representation of $\mathcal{M}$.

2. If there exists a regular representation $\rho$ of $\mathcal{M}$, then so is also $C$. 


\section{Proof}

$A d$ 1. Let $u v=w$ be in $\mathcal{M}$ and suppose we have two different 2-partitions $A B=C(w), A^{\prime} B^{\prime}=C(w)$, with $A \cong A^{\prime} \cong C(u), B \cong B^{\prime} \cong C(v)$. Suppose that $A$ is a proper initial segment of $A^{\prime}$. Since $A B$ corresponds to a 2-partition $u v$, there is a gap between $A$ and $B$. Since $A$ is a proper initial segment of $A^{\prime}$ the gap is in $A^{\prime}$. As every gap corresponds to a 2-partition of the preimage in $C$, there exists $y$ and $D$ such that $A^{\prime}=A D$ and $D \cong C(y)$. Hence $u=u y$, which is possible only if $y=\varepsilon$. But then $D$ is empty, which is a contradiction.

\section{Ad 2. Our strategy is}

(i) to construct an order preserving mapping $h: \operatorname{Supp}(\rho(w)) \rightarrow \operatorname{Supp}(C(w))$, for every $w \in M$, and then

(ii) to show that if $\iota: C(u) \rightarrow C(v)$ is an isomorphism, then for every $S \in \operatorname{Supp}(C(u))$ the fibers of $S$ and $\iota(S)$, as decorated orderings, are isomorphic, i.e.,

$$
h^{-1}(S) \cong h^{-1}(\iota(S)),
$$

or they are both empty.

Ad (i). Let $w \in M$, let $j \in \operatorname{Supp}(\rho(w))$. We define

$$
h(j)=\left\{(x, y, z) \mid \exists A, B, D\left(j \in B \text { and } \rho^{3}(x, y, z)=(A, B, D)\right)\right\} .
$$

One can readily verify that $h(j)$ is a wuf, and that $h$ is order preserving.

Ad (ii). Let $S \in \operatorname{Supp}(C(u))$ and $T \in \operatorname{Supp}(C(v))$ such that $T=\iota(S)$. Then $S$ and $T$ have the same decoration, which means that $S \sim T$. By definition, there exist 3-partitions $(x, y, z) \in S$ and $\left(x^{\prime}, y, z^{\prime}\right) \in T$ such that $S_{y}=T_{y}$. Let $\rho^{3}(x, y, z)=(A, B, D)$ and $\rho^{3}\left(x^{\prime}, y, z^{\prime}\right)=\left(A^{\prime}, B^{\prime}, D^{\prime}\right)$. Then $B \cong B^{\prime}$, as $\rho$ is a representation. Let us denote this isomorphism by $\kappa$.

Take an arbitrary 3 -partition $y_{1} y_{2} y_{3}=y$ and let

$$
\begin{aligned}
\rho^{5}\left(x, y_{1}, y_{2}, y_{3}, z\right) & =\left(A, B_{1}, B_{2}, B_{3}, D\right), \\
\rho^{5}\left(x^{\prime}, y_{1}, y_{2}, y_{3}, z^{\prime}\right) & =\left(A^{\prime}, B_{1}^{\prime}, B_{2}^{\prime}, B_{3}^{\prime}, D^{\prime}\right) .
\end{aligned}
$$

Then $B_{i} \cong B_{i}^{\prime}$, for $i=1,2,3$. By the regularity of $\rho$, the segments $B_{1}, B_{2}, B_{3}$ in $B$ and the segments $B_{1}^{\prime}, B_{2}^{\prime}, B_{3}^{\prime}$ in $B^{\prime}$ are uniquely determined by their isomorphism types, whence:

$$
\kappa\left(B_{i}\right)=B_{i}^{\prime} \text {, for } i=1,2,3 .
$$

The fiber $\iota^{-1}(S)$ is defined as the intersection of all segments $B_{2}$ that belong to 5-partitions $\left(x, y_{1}, y_{2}, y_{3}, z\right)$ such that $\left(y_{1}, y_{2}, y_{3}\right) \in S_{y}=T_{y}$. Similarly, the fiber $\iota^{-1}(T)$ is defined as the intersection of all segments $B_{2}^{\prime}$ that belong to 5 -partitions $\left(x^{\prime}, y_{1}, y_{2}, y_{3}, z^{\prime}\right)$ such that $\left(y_{1}, y_{2}, y_{3}\right) \in S_{y}=T_{y}$. According to (1), for all such 3-partitions, $\kappa:\left(B_{1}, B_{2}, B_{3}\right) \cong\left(B_{1}^{\prime}, B_{2}^{\prime}, B_{3}^{\prime}\right)$. Hence $\kappa$ is also an isomorphism of $\iota^{-1}(S)$ onto $\iota^{-1}(T)$, or both fibers are empty. 


\section{References}

[Bur05] John Burgess. Fixing Frege. Princeton Monographs in Philosophy. Princeton University Press, Princeton, 2005.

[Grz05] Andrzej Grzegorczyk. Undecidability without arithmetization. Studia Logica, 79:163-230, 2005.

[KT06] P. Komjáth and V. Totik. Problems and Theorems in Classical Set Theory. Springer-Verlag, New York, 2006.

[Sie58] W. Sierpiński. Cardinal and ordinal numbers, volume 34 of Monografie Matematyczne. Polska Akademia Nauk, Warszawa, 1958.

[Vis07] A. Visser. Growing commas -a study of sequentiality and concatenation. Logic Group Preprint Series 257, Department of Philosophy, Utrecht University, Heidelberglaan 8, 3584 CS Utrecht, http://www.phil.uu.nl/preprints/lgps/, 2007. 